\title{
Characterizations of $p$-superharmonic functions on metric spaces
}

\author{
by
}

ANDERS BJÖRN (Linköping)

\begin{abstract}
We show the equivalence of some different definitions of $p$-superharmonic functions given in the literature. We also provide several other characterizations of $p$ superharmonicity. This is done in complete metric spaces equipped with a doubling measure and supporting a Poincaré inequality. There are many examples of such spaces. A new one given here is the union of a line (with the one-dimensional Lebesgue measure) and a triangle (with a two-dimensional weighted Lebesgue measure). Our results also apply to Cheeger $p$-superharmonic functions and in the Euclidean setting to $\mathcal{A}$-superharmonic functions, with the usual assumptions on $\mathcal{A}$.
\end{abstract}

1. Introduction. Superharmonic functions are used as a tool to study harmonic functions. They are, e.g., used to define Perron solutions of the Dirichlet (i.e. boundary value) problem for harmonic functions. In connection with nonlinear $p$-harmonic functions on weighted $\mathbb{R}^{n}, p$-superharmonic functions were used in the monograph Heinonen-Kilpeläinen-Martio [7]. An important feature of the definition is that the bounded superharmonic functions (we drop $p$ from the notation from now on) are exactly the bounded lower semicontinuously regularized supersolutions (there are also unbounded superharmonic functions which are not supersolutions).

In metric spaces, Kinnunen-Martio [13] noticed that the definition from [7] was difficult to use (since at that time Theorem 3.7 below was not known). They proposed a different definition, which in the Euclidean case is equivalent to the definition in [7]. In connection with quasiminimizers, they gave a third definition in [14], and showed that a superharmonic function according to the third definition is also superharmonic according to the second definition.

In this paper we show that the definitions given in [7], [13] and [14] are equivalent. We also give several other characterizations of superharmonic-

2000 Mathematics Subject Classification: Primary 31C45; Secondary 31C05, 35J60, 49J27.

Key words and phrases: characterization, doubling measure, metric space, nonlinear, $p$-harmonic, Poincaré inequality, regular, superharmonic, superminimizer, supersolution. 
ity, and provide similar characterizations for hyperharmonic functions. (The obvious characterizations for subharmonic and hypoharmonic functions are left to the interested reader.)

There are many different examples of complete metric spaces equipped with a doubling measure satisfying a Poincaré inequality. Here are some of them:

(1) Unweighted and weighted Euclidean spaces (see the monograph by Heinonen-Kilpeläinen-Martio [7]).

(2) Riemannian manifolds with nonnegative Ricci curvature satisfy the $(1,2)$-Poincaré inequality (see Saloff-Coste [18]).

(3) Graphs (see Shanmugalingam [21]).

(4) The Heisenberg group $\mathbb{H}_{1}=\mathbb{C} \times \mathbb{R}$ with the Lebesgue measure and the metric

$$
d\left((z, t),\left(z^{\prime}, t^{\prime}\right)\right)=\left(\left|z-z^{\prime}\right|^{4}+\left(t-t^{\prime}+2 \operatorname{Im} \bar{z} z^{\prime}\right)^{2}\right)^{1 / 4}
$$

satisfies the (1,1)-Poincaré inequality (see Heinonen [6, Theorem 9.27]). Note that $\mathbb{H}_{1}$ is topologically 3 -dimensional but Ahlfors 4-regular, i.e. $\mu(B) \approx \operatorname{diam}(B)^{4}$ for balls $B$.

(5) For every $Q \geq 1$, Laakso [17] showed that there is an Ahlfors $Q$ regular space satisfying the $(1,1)$-Poincaré inequality.

(6) In Section 8, we construct an example where a line (with the onedimensional Lebesgue measure) is glued to a triangle (with a twodimensional weighted Lebesgue measure) so that the union satisfies the $(1,1)$-Poincaré inequality.

The results and proofs given in this paper also hold for Cheeger $p$ harmonic functions (see, e.g., Björn-Björn-Shanmugalingam [2] for a discussion). The results and proofs also hold for $\mathcal{A}$-harmonic functions as defined on p. 57 of Heinonen-Kilpeläinen-Martio [7], assuming that $\mathcal{A}$ satisfies the degenerate ellipticity conditions (3.3)-(3.7) on p. 56 of [7].

When this paper was almost ready, J. Björn [5] obtained Theorem 3.7, which improved Theorems 6.1 and 7.1.

Acknowledgements. The author is supported by the Swedish Research Council and Gustaf Sigurd Magnuson's fund of the Royal Swedish Academy of Sciences. This research started while the author was visiting the Department of Mathematical Analysis at the Charles University in Prague during the autumn 2003.

2. Notation and preliminaries. We assume throughout the paper that $X=(X, d, \mu)$ is a complete metric space endowed with a metric $d$ and a doubling measure $\mu$, i.e. there exists a constant $C>0$ such that for all balls $B=B\left(x_{0}, r\right):=\left\{x \in X: d\left(x, x_{0}\right)<r\right\}$ in $X$ (we make the convention 
that balls are nonempty and open),

$$
0<\mu(2 B) \leq C \mu(B)<\infty,
$$

where $\lambda B=B\left(x_{0}, \lambda r\right)$. We emphasize that the $\sigma$-algebra on which $\mu$ is defined is obtained by the completion of the Borel $\sigma$-algebra. We also assume that $1<p<\infty$ and that $\Omega \subset X$ is a nonempty open set. (At the end of this section we make one further assumption on $X$ that is kept in the rest of the paper.)

Note that some authors assume that $X$ is proper (i.e. closed bounded sets are compact) rather than complete, but, since $\mu$ is doubling, $X$ is proper if and only if it is complete.

A curve is a continuous mapping from an interval. We will in addition, throughout the paper, assume that every curve is nonconstant, compact and rectifiable. A curve can thus be parameterized by its arc length $d s$.

Definition 2.1. A nonnegative Borel function $g$ on $X$ is an upper gradient of an extended real-valued function $f$ on $X$ if for all curves $\gamma$ : $\left[0, l_{\gamma}\right] \rightarrow X$,

$$
\left|f(\gamma(0))-f\left(\gamma\left(l_{\gamma}\right)\right)\right| \leq \int_{\gamma} g d s
$$

whenever both $f(\gamma(0))$ and $f\left(\gamma\left(l_{\gamma}\right)\right)$ are finite, and $\int_{\gamma} g d s=\infty$ otherwise. If $g$ is a nonnegative measurable function on $X$ and if (2.1) holds for $p$-almost every curve, then $g$ is a $p$-weak upper gradient of $f$.

By saying that (2.1) holds for $p$-almost every curve we mean that it fails only for a curve family with zero $p$-modulus (see Definition 2.1 in Shanmugalingam [19]). It is implicitly assumed that $\int_{\gamma} g d s$ is defined (with a value in $[0, \infty])$ for $p$-almost every curve.

If $g \in L^{p}(X)$ is a $p$-weak upper gradient of $f$, then one can find a sequence $\left\{g_{j}\right\}_{j=1}^{\infty}$ of upper gradients of $f$ such that $g_{j} \rightarrow g$ in $L^{p}(X)$ (see Lemma 2.4 in Koskela-MacManus [16]).

If $f$ has an upper gradient in $L^{p}(X)$, then it has a minimal p-weak upper gradient $g_{f} \in L^{p}(X)$ in the sense that for every $p$-weak upper gradient $g \in L^{p}(X)$ of $f, g_{f} \leq g \mu$-a.e. (see Corollary 3.7 in Shanmugalingam [20]). The minimal $p$-weak upper gradient can be given by the formula

$$
g_{f}(x):=\inf _{g} \limsup _{r \rightarrow 0+} \underset{B(x, r)}{f} g d \mu,
$$

where $f_{A} g d \mu:=\mu(A)^{-1} \int_{A} g d \mu$ and the infimum is taken over all upper gradients $g \in L^{p}(X)$ of $f$ (see Lemma 2.3 in J. Björn [4]). See also BjörnBjörn [1, Section 3] for some further comments on $p$-weak upper gradients. 
Definition 2.2. We say that $X$ supports a weak $(1, q)$-Poincaré inequality if there exist constants $C>0$ and $\lambda \geq 1$ such that for all balls $B \subset X$, all measurable functions $f$ on $X$ and all upper gradients $g$ of $f$,

$$
f_{B}\left|f-f_{B}\right| d \mu \leq C \operatorname{diam}(B)\left(f_{\lambda B} g^{q} d \mu\right)^{1 / q},
$$

where $f_{B}:=f_{B} f d \mu$. If $\lambda=1$, then $X$ supports a $(1, q)$-Poincaré inequality.

In the above definition of Poincaré inequality we can equivalently assume that $g$ is a $q$-weak upper gradient- see the comments above. It is also equivalent to require that $(2.2)$ holds for all $f \in \operatorname{Lip}_{\mathrm{c}}(X)$ and all upper gradients $g \in \operatorname{Lip}_{\mathrm{c}}(X)$ of $f$ (see Keith [8, Theorem 2]). Here $\operatorname{Lip}_{\mathrm{c}}(A)=\{f \in \operatorname{Lip}(A)$ : supp $f \Subset A\}$, where $E \Subset A$ if $\bar{E}$ is a compact subset of $A$.

By the Hölder inequality it is easy to see that if $X$ supports a weak $(1, q)$-Poincaré inequality, then it supports a weak $(1, s)$-Poincaré inequality for every $s>q$.

Following Shanmugalingam [19], we define a version of Sobolev spaces on the metric space $X$.

Definition 2.3. Whenever $u \in L^{p}(X)$, let

$$
\|u\|_{N^{1, p}(X)}=\left(\int_{X}|u|^{p} d \mu+\inf _{g} \int_{X} g^{p} d \mu\right)^{1 / p},
$$

where the infimum is taken over all upper gradients of $u$. The Newtonian space on $X$ is the quotient space

$$
N^{1, p}(X)=\left\{u:\|u\|_{N^{1, p}(X)}<\infty\right\} / \sim,
$$

where $u \sim v$ if and only if $\|u-v\|_{N^{1, p}(X)}=0$.

The space $N^{1, p}(X)$ is a Banach space and a lattice (see Shanmugalingam [19]).

Definition 2.4. The capacity of a set $E \subset X$ is the number

$$
C_{p}(E)=\inf \|u\|_{N^{1, p}(X)}^{p},
$$

where the infimum is taken over all $u \in N^{1, p}(X)$ such that $u=1$ on $E$.

The capacity is countably subadditive. For this and other properties as well as equivalent definitions of the capacity we refer to KilpeläinenKinnunen-Martio [10] and Kinnunen-Martio [11], [12].

We say that a property regarding points in $X$ holds quasieverywhere (q.e.) if the set of points for which the property does not hold has capacity zero. The capacity is the correct gauge for distinguishing between two Newtonian functions. If $u \in N^{1, p}(X)$, then $u \sim v$ if and only if $u=v$ q.e. Moreover, Corollary 3.3 in Shanmugalingam [19] shows that if $u, v \in N^{1, p}(X)$ and $u=v \mu$-a.e., then $u \sim v$. 
If $X$ supports a weak $(1, p)$-Poincaré inequality, then Lipschitz functions are dense in $N^{1, p}(X)$ and the functions in $N^{1, p}(X)$ are quasicontinuous (see [19]). This means that in the Euclidean setting, $N^{1, p}\left(\mathbb{R}^{n}\right)$ is the refined Sobolev space as defined on p. 96 of Heinonen-Kilpeläinen-Martio [7].

To be able to compare the boundary values of Newtonian functions we need a Newtonian space with zero boundary values. We let

$$
N_{0}^{1, p}(\Omega)=\left\{\left.f\right|_{\Omega}: f \in N^{1, p}(X) \text { and } f=0 \text { on } X \backslash \Omega\right\} .
$$

One can replace the assumption " $f=0$ on $X \backslash \Omega$ " with " $f=0$ q.e. on $X \backslash \Omega$ " without changing the resulting space $N_{0}^{1, p}(\Omega)$. Note that if $C_{p}(X \backslash \Omega)=0$, then $N_{0}^{1, p}(\Omega)=N^{1, p}(\Omega)$.

We say that $f \in N_{\text {loc }}^{1, p}(\Omega)$ if $f \in N^{1, p}\left(\Omega^{\prime}\right)$ for every open $\Omega^{\prime} \Subset \Omega$.

We end this section by recalling some standard notation. We let $f_{+}=$ $\max \{f, 0\}$. By a continuous function we always mean a real-valued continuous function, whereas a semicontinuous function is allowed to be extended real-valued, i.e. to take values in the extended real line $\overline{\mathbb{R}}:=[-\infty, \infty]$.

In addition to the assumptions made at the beginning of this section, from now on we assume that $X$ supports a weak $(1, p)$-Poincaré inequality. By Keith-Zhong [9] it follows that $X$ supports a weak $(1, q)$-Poincaré inequality for some $q \in[1, p)$, which was earlier a standard assumption.

3. The obstacle problem. We follow Kinnunen-Martio [13] in making the following definition of the obstacle problem.

Let $V \subset X$ be a nonempty bounded open set with $C_{p}(X \backslash V)>0$. (If $X$ is unbounded then the condition $C_{p}(X \backslash V)>0$ is of course immediately satisfied.)

Definition 3.1. Let $f \in N^{1, p}(V)$ and $\psi: V \rightarrow \overline{\mathbb{R}}$. Then we define

$$
\mathcal{K}_{\psi, f}(V)=\left\{v \in N^{1, p}(V): v-f \in N_{0}^{1, p}(V) \text { and } v \geq \psi \mu \text {-a.e. in } V\right\} .
$$

Further, a function $u \in \mathcal{K}_{\psi, f}(V)$ is a solution of the $\mathcal{K}_{\psi, f}(V)$-obstacle problem if

$$
\int_{V} g_{u}^{p} d \mu \leq \int_{V} g_{v}^{p} d \mu \quad \text { for all } v \in \mathcal{K}_{\psi, f}(V) .
$$

Kinnunen-Martio [13, Theorem 3.2] showed that if $\mathcal{K}_{\psi, f}(V) \neq \emptyset$, then there is a solution of the $\mathcal{K}_{\psi, f}(V)$-obstacle problem, and this solution is unique up to equivalence in $N^{1, p}(V)$. They also showed (Theorem 5.1 in [13]) that if $u$ is a solution then its lower semicontinuous regularization $u^{*}(x)=$ $\operatorname{ess}_{\liminf } \rightarrow x u(y)$ is also a solution and this solution is the unique lower semicontinuously regularized solution. Furthermore, $u^{*}$ is (a)-superharmonic in $V$ (see Definition 4.1). If the obstacle $\psi$ is continuous they showed that $u^{*}$ is also continuous (see Theorem 5.5 in [13]). They actually considered 
continuous functions which are even allowed to be extended real-valued. We will need the following special case of their result. For $f \in N^{1, p}(V)$, define $H_{V} f$ to be the continuous solution of the $\mathcal{K}_{-\infty, f}(V)$-obstacle problem.

Proposition 3.2. Let $f \in N^{1, p}(V)$ be continuous. Then there is a continuous solution $u$ of the $\mathcal{K}_{f, f}(V)$-obstacle problem. Moreover, $u \geq f$ everywhere in $V$, and $\left.u\right|_{A}=H_{A} f$ for the open set $A=\{x \in V: u(x)>f(x)\}$.

(See Björn-Björn [1, Proposition 4.2] for a proof.)

A function $u$ is a superminimizer in $\Omega$ if it is a solution of the $\mathcal{K}_{u, u}\left(\Omega^{\prime}\right)$ obstacle problem for every nonempty open subset $\Omega^{\prime} \Subset \Omega$ with $C_{p}\left(X \backslash \Omega^{\prime}\right)>0$. Equivalently, a function $u \in N_{\text {loc }}^{1, p}(\Omega)$ is a superminimizer in $\Omega$ if for every nonempty open subset $\Omega^{\prime} \Subset \Omega$ and all nonnegative $\varphi \in N_{0}^{1, p}\left(\Omega^{\prime}\right)$, we have

$$
\int_{\Omega^{\prime}} g_{u}^{p} d \mu \leq \int_{\Omega^{\prime}} g_{u+\varphi}^{p} d \mu
$$

This is just a rephrasing apart from the case when $\Omega=X$ is bounded, but in the latter case it is easy to see that $u$ must be constant q.e. An equivalent definition was given in Kinnunen-Martio [14, Section 3].

A solution $u$ of the $\mathcal{K}_{\psi, f}(V)$-obstacle problem is a superminimizer in $V$. Conversely, if $u \in N^{1, p}(V)$ is a superminimizer, then $u$ is a solution of the $\mathcal{K}_{u, u}(V)$-obstacle problem. A function $u$ is a minimizer in $\Omega$ if it is a solution of the $\mathcal{K}_{-\infty, u}\left(\Omega^{\prime}\right)$-obstacle problem for every nonempty open subset $\Omega^{\prime} \Subset \Omega$ with $C_{p}\left(X \backslash \Omega^{\prime}\right)>0$, or equivalently if both $u$ and $-u$ are superminimizers in $\Omega$.

By Proposition 3.8 and Corollary 5.5 in Kinnunen-Shanmugalingam [15], a minimizer in $\Omega$ can be modified on a set of capacity zero so that it becomes locally Hölder continuous in $\Omega$. A p-harmonic function is a continuous minimizer. By Corollary 6.4 in [15], $p$-harmonic functions satisfy the strong maximum principle: If $u$ attains its minimum or maximum in some component $G$ of $\Omega$, then $\left.u\right|_{G}$ is constant.

The sum of two $p$-harmonic functions is, in general, not a $p$-harmonic function. Nevertheless, if $u$ is $p$-harmonic and $\alpha, \beta \in \mathbb{R}$, then $\alpha u+\beta$ is also $p$-harmonic.

If $f_{1}, f_{2} \in N^{1, p}(V)$ and $\left(f_{1}-f_{2}\right)_{+} \in N_{0}^{1, p}(V)$, then $H_{V} f_{1} \leq H_{V} f_{2}$. (This is a special case of Lemma 3.9.) It follows that for $f \in N^{1, p}(\bar{V}), H_{V} f$ only depends on $\left.f\right|_{\partial V}$. A Lipschitz function $f$ on $\partial V$ can be extended to a function $\tilde{f} \in \operatorname{Lip}(\bar{V})$ so that $f=\tilde{f}$ on $\partial V$. As $H_{V} \widetilde{f}$ does not depend on the choice of extension, we define $H_{V} f:=H_{V} \tilde{f}$.

We say that $u$ is $K$-quasisuperharmonic in $\Omega$ if it is K-quasisuperharmonic in the sense of Definition 7.1 in Kinnunen-Martio [14]. (Note that there is a misprint in [14]: the functions $v_{i}$ should be assumed to be $K$-quasisuperminimizers.) 
A function $u$ is $K$-quasihyperharmonic in $\Omega$ if in each component of $\Omega$ it is $K$-quasisuperharmonic or identically $\infty$.

Definition 3.3. Given a function $f: \partial V \rightarrow \overline{\mathbb{R}}$, let $\mathcal{U}_{f}(V)$ be the set of all (a)-superharmonic functions $u$ in $V$ (see Definition 4.1) bounded below such that

$$
\liminf _{V \ni y \rightarrow x} u(y) \geq f(x) \quad \text { for all } x \in \partial V .
$$

Define the upper Perron solution of $f$ by

$$
\bar{P}_{V} f(x)=\inf _{u \in \mathcal{U}_{f}(V)} u(x), \quad x \in V .
$$

The lower Perron solution is given analogously or by

$$
\underline{P}_{V} f=-\bar{P}_{V}(-f) \text {. }
$$

If $\bar{P}_{V} f=\underline{P}_{V} f$, then we let $P_{V} f=\bar{P}_{V} f$, and $f$ is said to be resolutive.

The comparison principle given by Kinnunen-Martio [13, Theorem 7.2], shows that $\underline{P}_{V} f \leq \bar{P}_{V} f$ for all functions $f$.

We now have two ways of solving the Dirichlet problem for $p$-harmonic functions. We need two results from Björn-Björn-Shanmugalingam [2]:

Theorem 3.4 (Theorem 6.1 in [2]). Let $f \in C(\partial V)$. Then $f$ is resolutive.

Theorem 3.5 (Theorem 5.1 in [2]). Let $f \in N^{1, p}(X)$. Then $P_{V} f=H_{V} f$ in $V$.

Note that since $H_{V} f$ is independent of which representative of $f$ we pick, also $P_{V} f$ is independent of the representative (when $f \in N^{1, p}(X)$ ).

Definition 3.6. A point $x_{0} \in \partial V$ is regular if

$$
\lim _{V \ni y \rightarrow x_{0}} H_{V} f(y)=f\left(x_{0}\right) \quad \text { for all } f \in \operatorname{Lip}(\partial V) .
$$

If $x_{0} \in \partial V$ is not regular, then it is irregular. The set $V$ is regular if every $x_{0} \in \partial V$ is regular.

Note that when we say that a set is regular we require that it is nonempty, open, bounded and has a complement with positive capacity. For equivalent characterizations of regular boundary points see Björn-Björn [1, Theorem 6.2].

From J. Björn [5] we need the following result.

Theorem 3.7. Assume that either $X$ is unbounded or $\Omega \neq X$. Then there exist regular sets $\Omega_{1} \Subset \Omega_{2} \Subset \cdots$ such that $\Omega=\bigcup_{j=1}^{\infty} \Omega_{j}$.

We also need the following results from Björn-Björn [1]. 
Proposition 3.8 (Corollary 5.4 in [1]). Assume that $V$ is regular. Let $f \in \operatorname{Lip}(\bar{V})$, and let $u$ be the continuous solution of the $\mathcal{K}_{f, f}(V)$-obstacle problem in $V$, and $u=f$ on $\partial V$. Then $u \in C(\bar{V})$.

Lemma 3.9 (Lemma 5.6 in [1]). Let $\psi_{j}: V \rightarrow \overline{\mathbb{R}}$ and $f_{j} \in N^{1, p}(V)$ be such that $\mathcal{K}_{\psi_{j}, f_{j}}(V) \neq \emptyset$, and let $u_{j}$ be the lower semicontinuously regularized solution of the $\mathcal{K}_{\psi_{j}, f_{j}}(V)$-obstacle problem, $j=1,2$. Assume that $\psi_{1} \leq \psi_{2}$ $\mu$-a.e. in $V$ and that $\left(f_{1}-f_{2}\right)_{+} \in N_{0}^{1, p}(V)$. Then $u_{1} \leq u_{2}$ in $V$.

4. Definitions of superharmonicity. The main purpose of this note is to obtain equivalent definitions of superharmonicity; in order to be able to study this we need to give a number of different definitions of superharmonicity.

One should observe that even though we give different definitions of superharmonicity, the underlining definitions of harmonicity and superminimizers are the same throughout.

Definition 4.1. Let $u: \Omega \rightarrow(-\infty, \infty]$ and consider the following statements:

(i) The function $u$ is lower semicontinuous in $\Omega$.

(ii) The function $u$ is not identically $\infty$ in any component of $\Omega$.

(iii.a) For every nonempty open set $\Omega^{\prime} \Subset \Omega$ with $C_{p}\left(X \backslash \Omega^{\prime}\right)>0$, and all functions $v \in C\left(\overline{\Omega^{\prime}}\right) \cap N^{1, p}\left(\Omega^{\prime}\right)$ such that $v \leq u$ on $\partial \Omega^{\prime}$, we have $H_{\Omega^{\prime}} v \leq u$ in $\Omega^{\prime}$.

(iii.b) For every nonempty open set $\Omega^{\prime} \Subset \Omega$ with $C_{p}\left(X \backslash \Omega^{\prime}\right)>0$, and all functions $v \in \operatorname{Lip}\left(\partial \Omega^{\prime}\right)$ such that $v \leq u$ on $\partial \Omega^{\prime}$, we have $H_{\Omega^{\prime}} v \leq u$ in $\Omega^{\prime}$.

(iii.c) For every nonempty open set $\Omega^{\prime} \Subset \Omega$ with $C_{p}\left(X \backslash \Omega^{\prime}\right)>0$, and all functions $v \in N_{\text {loc }}^{1, p}(\Omega)$ such that $v \leq u$ q.e. on $\partial \Omega^{\prime}$, we have $H_{\Omega^{\prime}} v \leq u$ in $\Omega^{\prime}$.

(iii.d) For every nonempty open set $\Omega^{\prime} \Subset \Omega$ with $C_{p}\left(X \backslash \Omega^{\prime}\right)>0$, and all functions $v \in C\left(\overline{\Omega^{\prime}}\right) \cap N^{1, p}\left(\Omega^{\prime}\right)$ such that $v \leq u$ on $\overline{\Omega^{\prime}}$, we have $w \leq u$ in $\Omega^{\prime}$, where $w$ is the continuous solution of the $\mathcal{K}_{v, v}\left(\Omega^{\prime}\right)$-obstacle problem.

(iii.e) For every nonempty open set $\Omega^{\prime} \Subset \Omega$ with $C_{p}\left(X \backslash \Omega^{\prime}\right)>0$, and all functions $v \in \operatorname{Lip}\left(\overline{\Omega^{\prime}}\right)$ such that $v \leq u$ on $\overline{\Omega^{\prime}}$, we have $w \leq u$ in $\Omega^{\prime}$, where $w$ is the continuous solution of the $\mathcal{K}_{v, v}\left(\Omega^{\prime}\right)$-obstacle problem.

(iii.f) For every nonempty open set $\Omega^{\prime} \Subset \Omega$ with $C_{p}\left(X \backslash \Omega^{\prime}\right)>0$, and all functions $v \in C\left(\overline{\Omega^{\prime}}\right)$ such that $v$ is $p$-harmonic in $\Omega^{\prime}$ and $v \leq u$ on $\partial \Omega^{\prime}$, we have $v \leq u$ in $\Omega^{\prime}$. 
(iii.g) For every nonempty open set $\Omega^{\prime} \Subset \Omega$ with $\Omega^{\prime} \neq \Omega$, and all functions $v \in C\left(\overline{\Omega^{\prime}}\right)$ such that $v$ is $p$-harmonic in $\Omega^{\prime}$ and $v \leq u$ on $\partial \Omega^{\prime}$, we have $v \leq u$ in $\Omega^{\prime}$.

For $j=\mathrm{a}, \ldots, \mathrm{g}$, we define $u$ to be $(j)$-superharmonic in $\Omega$ if $u$ satisfies conditions (i), (ii) and (iii. $j$ ). Furthermore, we define $u$ to be $(j)$-hyperharmonic in $\Omega$ if $u$ satisfies conditions (i) and (iii. $j$ ).

We will show that the different definitions of superharmonicity above are equivalent.

The definition of $(\mathrm{g})$-superharmonicity is the one given in HeinonenKilpeläinen-Martio [7, p. 131] in the weighted Euclidean setting.

Kinnunen-Martio [13, Section 7] gave the definition of (a)-superharmonicity in the metric space setting. They knew that the definitions of (g)superharmonicity and (a)-superharmonicity are equivalent in the weighted Euclidean setting (this was mentioned at a public lecture given by Kinnunen at the University of Michigan, Ann Arbor, 28 February 2002).

In Björn-Björn-Shanmugalingam [2] it was observed, after Definition 3.10, that (a)-superharmonicity is equivalent to (b)-superharmonicity.

In connection with quasisuperminimizers, Kinnunen-Martio [14] gave the definition of (d)-superharmonicity. They showed that (d)-superharmonicity implies (a)-superharmonicity.

We have given special care to correctly handle the case $X=\Omega$ bounded.

REMARK 4.2. Let us make some observations for $j \in\{\mathrm{a}, \ldots, \mathrm{g}\}$.

(1) If $u$ is $p$-harmonic in $\Omega$, then $u$ is $(j)$-superharmonic in $\Omega$.

(2) The assumption "for every nonempty open set $\Omega^{\prime} \Subset \Omega$ " can be replaced by "for every domain $\Omega^{\prime} \Subset \Omega$ ". In one direction this is trivial and in the other it follows by arguing in each component of $\Omega^{\prime}$ separately. (A domain is by definition a nonempty open connected set.)

(3) Let $\Omega^{\prime} \Subset \Omega$. If $X$ is unbounded, then it is immediate that $C_{p}\left(X \backslash \Omega^{\prime}\right)$ is positive; similarly if $\Omega \neq X$, then there are $x \in X \backslash \Omega$ and $r>0$ so that $B(x, r) \subset X \backslash \Omega^{\prime}$, hence $C_{p}\left(X \backslash \Omega^{\prime}\right)>0$. Thus the condition $C_{p}\left(X \backslash \Omega^{\prime}\right)>0$ is redundant unless $X=\Omega$ is bounded, but in this case we need to have the obstacle problem uniquely soluble (and the operator $H_{\Omega^{\prime}}$ well defined), which is essential in (iii.a)-(iii.e). Alternatively one can formulate these definitions in a manner similar to (i) in Theorem 6.1 , or, in view of (5) below, just say that if $X$ is bounded, then $u$ is $(j)$-superharmonic on $X$ if $u$ is constant.

(4) If the condition $\Omega^{\prime} \neq \Omega$ were omitted from (iii.g) and $X$ were bounded, then, as all constant functions are $p$-harmonic, no function would be (g)-superharmonic on $X$, which would contradict (1).

(5) It follows from the maximum principle for $p$-harmonic functions and for the obstacle problem that $u \equiv k \in \mathbb{R}$ is $(j)$-superharmonic. Conversely, if $X$ is bounded, then all $(j)$-superharmonic functions on $X$ are constant. To 
see this, let $u$ be (d)-superharmonic on $X$. Let $m=\inf _{X} u$ and $M=\sup _{X} u$. Let $\delta<M$ be arbitrary and $x \in X$ be so that $u(x)>\delta$. Since $u$ is lower semicontinuous there is a ball $B=B(x, r)$ such that $u>\delta$ in $2 B$. Let $v \in \operatorname{Lip}(X)$ be such that $v=\delta$ in $B, v=m$ on $X \backslash 2 B$ and $m \leq v \leq \delta$ on $X$; then $v \leq u$ on $X$. Let $w$ be the continuous solution of the $\mathcal{K}_{v, v}(X \backslash \bar{B})$-obstacle problem. By Lemma 3.9 and the maximum principle for $p$-harmonic functions,

$$
u \geq w \geq H_{X \backslash \bar{B}} v \geq \delta \quad \text { in } X \backslash \bar{B} .
$$

Thus $u \geq \delta$ on $X$, and since $\delta<M$ was arbitrary, $u \equiv M<\infty$. The proofs for $j \neq \mathrm{d}$ are similar or easier.

(6) If $u$ and $v$ are (j)-superharmonic in $\Omega, a \geq 0$ and $b \in \mathbb{R}$, then $\min \{u, v\}$ and $a u+b$ are $(j)$-superharmonic in $\Omega$.

(7) A function $u$ satisfying (ii) is (j)-superharmonic in $\Omega$ if and only if $\min \{u, k\}$ is $(j)$-superharmonic in $\Omega$ for all $k \in \mathbb{R}$. In one direction this follows from (5) and (6). In the other direction, if $j \neq \mathrm{c}$, it follows since continuous functions are bounded on compact sets, and if $j=\mathrm{c}$ it will follow from the characterization in Theorem 6.1.

(8) Let $\Omega_{1} \subset \Omega_{2} \subset \cdots$ be an increasing sequence of open sets such that $\Omega=\bigcup_{k=1}^{\infty} \Omega_{k}$. Then a function is $(j)$-superharmonic in $\Omega$ if and only if it is $(j)$-superharmonic in $\Omega_{k}$ for every $k$. (This follows from a compactness argument.)

(9) A function is $(j)$-superharmonic in $\Omega$ if and only if it is $(j)$-superharmonic in every component of $\Omega$.

(10) A function is $(j$ )-hyperharmonic in $\Omega$ if and only if in every component of $\Omega$ it is either identically $\infty$ or $(j)$-superharmonic.

(11) There are obvious counterparts for subharmonic and hypoharmonic functions.

5. Functions that are (f)-superharmonic. In this section we prove the results for (f)-superharmonic functions needed in the proof of Theorem 6.1. The proofs in this section are fairly close to those in Section 7 of Kinnunen-Martio [13]. The author has been unable to follow the proof of Lemma 7.16 in [13], however, Kinnunen-Martio made all the preparations for a correct proof. For the reader who so wishes it is an easy task to modify the proof of Lemma 5.4 below to give an alternative proof of Lemma 7.16 in [13].

THEOREM 5.1. Let $u$ be (f)-superharmonic in $\Omega$ and let $\Omega^{\prime} \Subset \Omega$ be regular. Then there is an increasing sequence of continuous superminimizers $\left\{u_{j}\right\}_{j=1}^{\infty}$ in $\Omega^{\prime}$ such that $u(x)=\lim _{j \rightarrow \infty} u_{j}(x)$ for every $x \in \Omega^{\prime}$.

Proof. Since $u$ is lower semicontinuous in $\Omega$, there is an increasing sequence $\left\{\varphi_{j}\right\}_{j=1}^{\infty}$ of Lipschitz functions on $\overline{\Omega^{\prime}}$ such that $u=\lim _{j \rightarrow \infty} \varphi_{j}$ 
on $\overline{\Omega^{\prime}}$. Let $u_{j}$ be the continuous solution of the $\mathcal{K}_{\varphi_{j}, \varphi_{j}}\left(\Omega^{\prime}\right)$-obstacle problem in $\Omega^{\prime}$, and $u_{j}=\varphi_{j}$ on $\partial \Omega^{\prime}$. By Proposition 3.8, $u_{j} \in C\left(\overline{\Omega^{\prime}}\right)$. Let $A_{j}=\left\{x \in \Omega^{\prime}: u_{j}(x)>\varphi_{j}(x)\right\}$. By Proposition $3.2, A_{j}$ is an open set, $u_{j}=H_{A_{j}} \varphi_{j}$ is $p$-harmonic in $A_{j}$ and $u_{j}=\varphi_{j} \leq u$ on $\partial A_{j}$. Since $u$ is (f)-superharmonic, it follows that $u_{j} \leq u$ in $A_{j}$. Thus

$$
u=\lim _{j \rightarrow \infty} \varphi_{j} \leq \lim _{j \rightarrow \infty} u_{j} \leq u \quad \text { in } \Omega^{\prime},
$$

and hence $u=\lim _{j \rightarrow \infty} u_{j}$ in $\Omega^{\prime}$. That the sequence $\left\{u_{j}\right\}_{j=1}^{\infty}$ is increasing follows from Lemma 3.9.

Corollary 5.2. Let $u$ be (f)-superharmonic in $\Omega$ and let $\Omega^{\prime} \Subset \Omega$ be regular. If $u$ is locally bounded in $\Omega^{\prime}$, then $u$ is a superminimizer in $\Omega^{\prime}$.

Proof. By Theorem 5.1, $u$ is a limit of an increasing sequence of superminimizers. It follows from Theorem 6.1 in [13] that $u$ is a superminimizer in $\Omega^{\prime}$.

Lemma 5.3. Let $u$ be (f)-superharmonic in $\Omega$ and let $\Omega^{\prime} \Subset \Omega$ be regular. Then for every ball $B$ such that $u \geq 0$ in $6 B \subset \Omega^{\prime}$ we have

$$
\left(f_{B} u^{\sigma} d \mu\right)^{1 / \sigma} \leq \inf _{3 B} u
$$

where $c<\infty$ and $\sigma>0$ only depend on $p$ and the constants in the doubling condition and the Poincaré inequality.

Proof. Construct the sequence $\left\{u_{j}\right\}_{j=1}^{\infty}$ as in the proof of Theorem 5.1. In the construction we can assume that $\varphi_{j} \geq 0$ in $5 B$, and hence that $0 \leq$ $u_{j} \leq u$ in $5 B$. Using Lemma 4.7 of [13], and the fact that $u_{j}$ is continuous, we see that

$$
\left(f u_{B}^{\sigma} d \mu\right)^{1 / \sigma} \leq c \underset{3 B}{\operatorname{essinf}} u_{j}=c \inf _{3 B} u_{j} \leq c \inf _{3 B} u .
$$

Since $u_{j}$ increases to $u$, the monotone convergence theorem shows that

$$
\left(f_{B} u^{\sigma} d \mu\right)^{1 / \sigma} \leq c \inf _{3 B} u .
$$

Lemma 5.4. Let $u$ be (f)-superharmonic in $\Omega, \Omega^{\prime} \Subset \Omega$ be regular and $\Omega^{\prime \prime} \subset \Omega^{\prime}$ be open. Assume that $u=0 \mu$-a.e. in $\Omega^{\prime \prime}$. Then $u=0$ everywhere in $\Omega^{\prime \prime}$.

Proof. Let $x \in \Omega^{\prime \prime}$. Since $u$ is lower semicontinuous,

$$
u(x) \leq \liminf _{y \rightarrow x} u(y) \leq 0 .
$$

Let $\varepsilon>0$. Again since $u$ is lower semicontinuous, there is a ball $B=$ 
$B(x, r) \subset 6 B \subset \Omega^{\prime \prime}$ such that

$$
u(y) \geq u(x)-\varepsilon \quad \text { for } y \in 6 B .
$$

Then $v=u-u(x)+\varepsilon$ is an (f)-superharmonic function in $\Omega$ such that $v \geq 0$ in $6 B$ and $v=-u(x)+\varepsilon \mu$-a.e. in $6 B$. So by Lemma 5.3 ,

$$
-u(x) \leq-u(x)+\varepsilon=\left(f_{B} v^{\sigma} d \mu\right)^{1 / \sigma} \leq \inf _{3 B} v \leq c v(x)=c \varepsilon .
$$

Letting $\varepsilon \rightarrow 0+$ gives $-u(x) \leq 0$, i.e. $u(x)=0$.

THEOREM 5.5. Let $u$ be (f)-superharmonic in $\Omega$ and let $\Omega^{\prime} \Subset \Omega$ be regular. Then

$$
u(x)=\operatorname{ess} \lim _{y \rightarrow x} \inf u(y) \quad \text { for every } x \in \Omega^{\prime} .
$$

Proof. Let $x \in \Omega^{\prime}$. Since $u$ is lower semicontinuous,

$$
u(x) \leq \liminf _{y \rightarrow x} u(y) \leq \operatorname{ess} \lim _{y \rightarrow x} \inf u(y)=: \lambda .
$$

Let $a<\lambda$. Then there is a ball $B=B(x, r) \subset \Omega^{\prime}$ such that

$$
u(y)>a \quad \text { for } \mu \text {-a.e. } y \in B \text {. }
$$

Let $v=\min \{u-a, 0\}$, an (f)-superharmonic function in $\Omega$ such that $v=0$ $\mu$-a.e. in $B$. By Lemma 5.4, $v=0$ everywhere in $B$, and thus $u(x) \geq a$. Letting $a \rightarrow \lambda$ shows that $u(x)=\lambda$.

\section{Characterizations of superharmonicity}

Theorem 6.1. Let $u: \Omega \rightarrow(-\infty, \infty]$. Then the following are equivalent:

(a) $u$ is (a)-superharmonic in $\Omega$;

(b) $u$ is (b)-superharmonic in $\Omega$;

(g) $u$ is (g)-superharmonic in $\Omega$;

(h) $u$ is 1-quasisuperharmonic in $\Omega$;

(i) $u$ is (d)-superharmonic in $\Omega^{\prime}$ for every nonempty open set $\Omega^{\prime} \subset \Omega$ with $C_{p}\left(X \backslash \Omega^{\prime}\right)>0$;

(j) $u$ is not identically $\infty$ in any component of $\Omega$, and $\min \{u, k\}$ is (d)-superharmonic in $\Omega$ for every $k \in \mathbb{R}$;

(k) $u$ is not identically $\infty$ in any component of $\Omega$, and $\min \{u, k\}$ is a lower semicontinuously regularized superminimizer in $\Omega$ for every $k \in \mathbb{R}$

(1) $u$ is not identically $\infty$ in any component of $\Omega$, and for every $k \in \mathbb{R}, u_{k}:=\min \{u, k\}$ is a superminimizer in $\Omega$ such that $u_{k}(x)=$ $\lim _{r \rightarrow 0+} f_{B(x, r)} u_{k} d \mu$ for $x \in \Omega$. 
Of course, in (i) and (j) we can replace d by a, b, c, e, f or g once this theorem has been proved.

Proof. (d) $\Rightarrow$ (i). This is trivial.

(i) $\Rightarrow(\mathrm{d})$. If $\Omega=X$ is bounded, then the proof given in Remark 4.2(5) actually only uses the fact that $u$ is (d)-superharmonic in $X \backslash \bar{B}$ for every ball $B \subset X$, and thus $u$ is constant and we are done. Otherwise, we can find $\Omega_{1} \Subset \Omega_{2} \Subset \cdots$ such that $\Omega=\bigcup_{j=1}^{\infty} \Omega_{j}$. As $C_{p}\left(X \backslash \Omega_{j}\right)>0, u$ is (d)-superharmonic in $\Omega_{j}$ for every $j$, and thus by Remark 4.2(8), $u$ is (d)superharmonic in $\Omega$.

$(d) \Rightarrow(a)$. This is Lemma 8.5 in Kinnunen-Martio [14]. We however give an alternative proof here: If $\Omega=X$ is bounded, then this follows from Remark 4.2(5). Otherwise, we can find $\Omega_{1} \Subset \Omega_{2} \Subset \cdots$ such that $\Omega=\bigcup_{j=1}^{\infty} \Omega_{j}$. For every $j$, by Lemma 8.2 in [14] there exists an increasing sequence $\left\{u_{j, k}\right\}_{k=1}^{\infty}$ of continuous superminimizers in $\Omega_{j}$ such that $u=\lim _{k \rightarrow \infty} u_{j, k}$ everywhere in $\Omega_{j}$. By Proposition 7.4 in Kinnunen-Martio [13], $u_{j, k}$ is (a)superharmonic in $\Omega_{j}$. Hence, by Lemma 7.1 in [13], $u$ is (a)-superharmonic in $\Omega_{j}$. Finally, by Remark 4.2(8), $u$ is (a)-superharmonic in $\Omega$.

(a) $\Rightarrow(\mathrm{c})$. Let $\Omega^{\prime} \Subset \Omega$ be a nonempty open set with $C_{p}\left(X \backslash \Omega^{\prime}\right)>0$ and let $v \in N_{\text {loc }}^{1, p}(\Omega)$ be such that $v \leq u$ q.e. on $\partial \Omega^{\prime}$. Let $\eta \in \operatorname{Lip}_{\mathrm{c}}(\Omega)$ be 1 on $\overline{\Omega^{\prime}}$ and

$$
\widetilde{v}= \begin{cases}\min \{v, u\} & \text { on } \partial \Omega^{\prime}, \\ \eta v & \text { in } X \backslash \partial \Omega^{\prime} .\end{cases}
$$

Then $\widetilde{v}=\eta v$ q.e. on $X$, and as $\eta v \in N^{1, p}(X)$ also $\widetilde{v} \in N^{1, p}(X)$. Moreover $\widetilde{v} \leq u$ on $\partial \Omega^{\prime}$. Since $u$ is lower semicontinuous it is bounded from below on $\overline{\Omega^{\prime}}$. Furthermore, $\liminf _{\Omega^{\prime} \ni y \rightarrow x} u(y) \geq u(x) \geq \widetilde{v}(x)$ for $x \in \partial \Omega^{\prime}$, from which it follows, by definition, that $u \in \mathcal{U}_{\widetilde{v}}\left(\Omega^{\prime}\right)$. Using Theorem 3.5, we see that

$$
u \geq P_{\Omega^{\prime}} \widetilde{v}=H_{\Omega^{\prime}} \widetilde{v}=H_{\Omega^{\prime}} v \quad \text { in } \Omega^{\prime} .
$$

$(\mathrm{c}) \Rightarrow(\mathrm{b})$. This follows since any Lipschitz function on $\partial \Omega^{\prime}$ can be extended to a Lipschitz function in $\Omega$, and $\operatorname{Lip}(\Omega) \subset N_{\text {loc }}^{1, p}(\Omega)$.

(b) $\Rightarrow(\mathrm{g})$. If $\Omega=X$ is bounded, then $u$ is constant by Remark 4.2(5) and thus $u$ is (g)-superharmonic. Otherwise, let $\Omega^{\prime} \Subset \Omega$ be a nonempty open set. Then $C_{p}\left(X \backslash \Omega^{\prime}\right)>0$. Let $v \in C\left(\overline{\Omega^{\prime}}\right)$ be $p$-harmonic in $\Omega^{\prime}$ and such that $v \leq u$ on $\partial \Omega^{\prime}$. By Corollary 6.2 in Björn-Björn-Shanmugalingam [2], $v=P_{\Omega^{\prime}} v$ in $\Omega^{\prime}$. Let $\varepsilon>0$. Then there exists $v^{\prime} \in \operatorname{Lip}\left(\overline{\Omega^{\prime}}\right) \subset N^{1, p}\left(\overline{\Omega^{\prime}}\right)$ such that $v^{\prime} \leq v \leq v^{\prime}+\varepsilon$ on $\overline{\Omega^{\prime}}$. Since $v^{\prime} \leq u$ on $\partial \Omega^{\prime}$ and $u$ is (b)-superharmonic, $P_{\Omega^{\prime}} v^{\prime}=H_{\Omega^{\prime}} v^{\prime} \leq u$ in $\Omega^{\prime}$. By the comparison principle we have $v=P_{\Omega^{\prime}} v \leq$ $P_{\Omega^{\prime}} v^{\prime}+\varepsilon \leq u+\varepsilon$ in $\Omega^{\prime}$. Letting $\varepsilon \rightarrow 0+$ completes the proof of this implication.

$(\mathrm{g}) \Rightarrow(\mathrm{f})$. This is trivial. 
(f) $\Rightarrow(\mathrm{k})$. If $\Omega=X$ is bounded, then $u$ is constant by Remark $4.2(5)$ and thus (k) holds. Otherwise, by Theorem 3.7, there exist regular sets $\Omega_{1} \Subset \Omega_{2} \Subset \cdots$ such that $\Omega=\bigcup_{j=1}^{\infty} \Omega_{j}$. Let $k \in \mathbb{R}$ and $u_{k}=\min \{u, k\}$. By Corollary 5.2, $u_{k}$ is a superminimizer in $\Omega_{j}$ for every $j$, from which it follows that $u_{k}$ is a superminimizer in $\Omega$. By Theorem 5.5, $u_{k}$ is lower semicontinuously regularized in $\Omega_{j}$ for every $j$ and thus in all of $\Omega$.

$(\mathrm{k}) \Rightarrow(\mathrm{j})$. This follows directly from Lemma 8.3 in Kinnunen-Martio [14]. (For the case $C_{p}(X \backslash \Omega)=0$ use the fact that we have already shown that $(\mathrm{d}) \Leftrightarrow(\mathrm{i})$.)

$(\mathrm{j}) \Rightarrow(\mathrm{d})$. This follows from Remark 4.2(7).

(d) $\Rightarrow(\mathrm{e})$. This is trivial.

(e) $\Rightarrow(\mathrm{d})$. Let $\Omega^{\prime} \Subset \Omega$ be a nonempty open set with $C_{p}\left(X \backslash \Omega^{\prime}\right)>0$. Let further $v \in C\left(\overline{\Omega^{\prime}}\right) \cap N^{1, p}\left(\Omega^{\prime}\right)$ be such that $v \leq u$ on $\overline{\Omega^{\prime}}$. Let $\varepsilon>0$. Then there exists $v^{\prime} \in \operatorname{Lip}\left(\overline{\Omega^{\prime}}\right) \subset N^{1, p}\left(\overline{\Omega^{\prime}}\right)$ such that $v^{\prime} \leq v \leq v^{\prime}+\varepsilon$ on $\overline{\Omega^{\prime}}$. Let $w$ and $w^{\prime}$ be the continuous solutions of the $\mathcal{K}_{v, v}\left(\Omega^{\prime}\right)$ - and $\mathcal{K}_{v^{\prime}, v^{\prime}}\left(\Omega^{\prime}\right)$-obstacle problems, respectively. Since $v^{\prime} \leq u$ on $\overline{\Omega^{\prime}}$ and $u$ is (e)-superharmonic, $w^{\prime} \leq$ $u$ in $\Omega^{\prime}$. By Lemma 3.9, $w \leq w^{\prime}+\varepsilon \leq u+\varepsilon$ in $\Omega^{\prime}$. Letting $\varepsilon \rightarrow 0+$ completes the proof of this implication.

$(\mathrm{k}) \Leftrightarrow(\mathrm{h})$. This is Theorem 7.10 in Kinnunen-Martio [14] (which unfortunately is not correctly stated, but should be stated in the form of the equivalence we want here).

$(\mathrm{k}) \Leftrightarrow(\mathrm{l})$. This follows from Remark 5.4 in Kinnunen-Martio [13], which says that

$$
\text { ess } \liminf _{y \rightarrow x} v(y)=\lim _{r \rightarrow 0+} \underset{B(x, r)}{f} v d \mu, \quad x \in \Omega,
$$

for any bounded superminimizer $v$ in $\Omega$.

\section{Characterizations of hyperharmonicity}

TheOREm 7.1. Let $u: \Omega \rightarrow(-\infty, \infty]$. Then the following are equivalent:

(a) $u$ is (a)-hyperharmonic in $\Omega$;

(b) $u$ is (b)-hyperharmonic in $\Omega$;

(g) $u$ is (g)-hyperharmonic in $\Omega$;

(h) $u$ is 1-quasihyperharmonic in $\Omega$;

(i) $u$ is (d)-hyperharmonic in $\Omega^{\prime}$ for every nonempty open set $\Omega^{\prime} \subset \Omega$ with $C_{p}\left(X \backslash \Omega^{\prime}\right)>0$;

(j) $\min \{u, k\}$ is (a)-superharmonic in $\Omega$ for every $k \in \mathbb{R}$;

(k) $\min \{u, k\}$ is a lower semicontinuously regularized superminimizer in $\Omega$ for every $k \in \mathbb{R}$;

(1) for every $k \in \mathbb{R}, u_{k}:=\min \{u, k\}$ is a superminimizer in $\Omega$ such that $u_{k}(x)=\lim _{r \rightarrow 0+} f_{B(x, r)} u_{k} d \mu$ for $x \in \Omega$. 
Proof. Assume first that $\Omega$ is connected. If $u \equiv \infty$ in $\Omega$, then all statements are true. On the other hand, if $u \not \equiv \infty$ in $\Omega$, then the result follows directly from Theorem 6.1.

Assume next that $\Omega$ is arbitrary. Then we argue in each component separately and obtain the full result using Remark 4.2(10).

8. A line joined to a triangle. In this section we give an example of a space $X$ satisfying our standard assumptions including the $(1,1)$-Poincaré inequality, such that the local dimension of the space is different at different parts of the space.

We let $X_{1}=[-1,0], X_{2}=\{z \in \mathbb{C}: 0 \leq \operatorname{Re} z \leq 1$ and $|\arg z| \leq \pi / 4\}$ (other angles can be obtained by applying a linear transformation) and $X=$ $X_{1} \cup X_{2}$. (We use complex notation to simplify some expressions.) Further, let $\left.\mu\right|_{X_{1}}$ be the one-dimensional Lebesgue measure, and $\left.d \mu\right|_{X_{2}}=|z|^{-1} d m$, where $m$ is the two-dimensional Lebesgue measure, i.e. $\left.d \mu\right|_{X_{2}}=d r d \theta$ in polar coordinates.

We need to prove that $\mu$ is doubling and that $X$ satisfies the $(1,1)$ Poincaré inequality. We start with the Poincaré inequality.

Let $u$ be a measurable function on $X$ and $g$ an upper gradient of $u$. Let $B=B\left(x_{0}, r_{0}\right) \subset X$ be a ball. We want to prove that there is a constant $a_{B}$ such that

$$
\int_{B}\left|u-a_{B}\right| d \mu \leq C \operatorname{diam}(B) \int_{B} g d \mu,
$$

for if this holds then

$$
\begin{aligned}
f_{B}\left|u-u_{B}\right| d \mu & \leq f_{B}\left|u-a_{B}\right| d \mu+\left|u_{B}-a_{B}\right| \\
& \leq f_{B}\left|u-a_{B}\right| d \mu+f_{B}\left|u-a_{B}\right| d \mu \\
& =2 f_{B}\left|u-a_{B}\right| d \mu \leq 2 C \operatorname{diam}(B) f_{B} g d \mu .
\end{aligned}
$$

Assume first that $B \cap X_{1} \neq \emptyset$. We let $T_{0}=\inf \left\{x \in B \cap X_{1}\right\}, T_{1}=$ $\sup \left\{x \in B \cap X_{1}\right\}$ and $a_{B}=u\left(T_{1}\right)$. Then

$$
\begin{aligned}
\int_{B \cap X_{1}}\left|u-a_{B}\right| d \mu & \leq \int_{T_{0}}^{T_{1}} \int_{t}^{T_{1}} g(\tau) d \tau d t \leq\left|T_{1}-T_{0}\right| \int_{T_{0}}^{T_{1}} g(\tau) d \tau \\
& \leq \operatorname{diam}(B) \int_{B \cap X_{1}} g d \mu .
\end{aligned}
$$


Thus we have proved (8.1) in the case when $B \subset X_{1}$. We therefore assume that $0 \in B$. Furthermore, let $r_{\theta}=\sup \left\{r \geq 0: r e^{i \theta} \in B \cap X_{2}\right\}$. Then

$$
\begin{aligned}
\int_{B \cap X_{2}}\left|u-a_{B}\right| d \mu & =\int_{-\pi / 4}^{\pi / 4} \int_{0}^{r_{\theta}}\left|u\left(r e^{i \theta}\right)-u(0)\right| d r d \theta \\
& \leq \int_{-\pi / 4}^{\pi / 4} \int_{0}^{r_{\theta}} \int_{0}^{r} g\left(\varrho e^{i \theta}\right) d \varrho d r d \theta \\
& \leq \int_{-\pi / 4}^{\pi / 4} r_{\theta} \int_{0}^{r_{\theta}} g\left(\varrho e^{i \theta}\right) d \varrho d \theta \leq \operatorname{diam}(B) \int_{B \cap X_{2}} g d \mu .
\end{aligned}
$$

Combining this with (8.2) gives

$$
\int_{B}\left|u-a_{B}\right| d \mu \leq \operatorname{diam}(B) \int_{B} g d \mu .
$$

It remains to consider the case $B=B(x, r) \subset X_{2} \backslash\{0\}, x \in X_{2}$. Let $v=\left.u\right|_{X_{2}}$ and extend $v$ by reflections to all of $\mathbb{C}$, i.e. let $v(z)=v(w)$ if $z \sim w$, where $\sim$ is the equivalence class on $\mathbb{C}$ defined by saying that

$a+i b \sim-a-i b \sim b+i a, \quad \pm 1+a+i b \sim \pm 1-a+i b, \quad a \pm i+i b \sim a \pm i-i b$, $a, b \in \mathbb{R}$. Note that for every $z \in \mathbb{C}$, there is a unique $w \in X_{2}$ such that $z \sim w$. We extend the measure $\left.d \mu\right|_{X_{2}}$ to $d \widetilde{\mu}=d r d \theta$ on $\mathbb{C}$. It is straightforward to check that $\widetilde{\mu}$ is a Muckenhoupt $A_{1}$ weight and therefore $\widetilde{\mu}$ is doubling and satisfies a $(1,1)$-Poincaré inequality (see, e.g., J. Björn [3, Theorem 4]). Let now $g$ be an upper gradient of $u$. Then $\widetilde{g}$, defined by $\widetilde{g}=g$ on $X_{2}$ and $\widetilde{g}(z)=\widetilde{g}(w)$ if $z \sim w$, is an upper gradient of $v$ on $\mathbb{C}$. Let $\widetilde{B}=B(x, r)$ as a ball in $\mathbb{C}$. Since $0 \notin B$, for every $z \in B$ there are at most eight $w \in \widetilde{B}$ such that $w \sim z$. Moreover, for every $w \in \widetilde{B}$, there exists $z \in B$ with $|z| \leq|w|$. Since $d \widetilde{\mu}(z)=w(z) d m$, where $w(z)=|z|^{-1}$ decreases with $|z|$ and $m$ is the two-dimensional Lebesgue measure, we see that

$$
\int_{B}\left|u-v_{\widetilde{B}}\right| d \mu \leq \int_{\widetilde{B}}\left|v-v_{\widetilde{B}}\right| d \mu \leq C r \int_{\widetilde{B}} \widetilde{g} d \mu \leq 8 C r \int_{B} g d \mu .
$$

We have thus proved the $(1,1)$-Poincaré-inequality.

Let us now turn to the doubling property, and start with the case $B=$ $B(x, r) \subset X$, where $x \in X_{2}$ and $r<3$. Let also $\widetilde{B}=B(x, r)$ as a subset of $\mathbb{C}$. For every $z \in B \cap X_{2}$ there are at most eighty $w \in \widetilde{B}$ such that $w \sim z$. Since $\widetilde{\mu}$ is doubling, we have

$$
\mu\left(2 B \cap X_{2}\right) \leq \widetilde{\mu}(2 \widetilde{B}) \leq C \mu(\widetilde{B}) \leq 80 C \mu(B) .
$$


If $2 B \cap X_{1} \neq \emptyset$, then there is $0 \leq \theta^{\prime} \leq \frac{1}{4} \pi$ such that

$$
\left\{\varrho e^{i \theta}: 0 \leq \varrho \leq r \text { and } \theta^{\prime}-\frac{1}{4} \pi \leq \arg z \leq \theta^{\prime}\right\} \subset 2 B \cap X_{2} .
$$

It follows that

$$
\mu\left(2 B \cap X_{1}\right) \leq 2 r \leq \frac{8}{\pi} \mu\left(2 B \cap X_{2}\right),
$$

and together with (8.3) this shows that $\mu$ is doubling for balls $B$ with center in $X_{2}$.

Finally, consider $B=B(x, r)$ with $x \in X_{1}, r<3$. Then

$$
\mu(2 B) \leq 2 r+\int_{-\pi / 4}^{\pi / 4} \int_{0}^{2 r} d \varrho d \theta \leq 6 r \leq 18 \mu\left(B \cap X_{1}\right) \leq 18 \mu(B) .
$$

We have thus shown that $\mu$ is doubling.

\section{References}

[1] A. Björn and J. Björn, Boundary regularity for p-harmonic functions and solutions of the obstacle problem, preprint, Linköping, 2004.

[2] A. Björn, J. Björn and N. Shanmugalingam, The Perron method for p-harmonic functions, J. Differential Equations 195 (2003), 398-429.

[3] J. Björn, Poincaré inequalities for powers and products of admissible weights, Ann. Acad. Sci. Fenn. Math. 26 (2001), 175-188.

[4] -, Boundary continuity for quasiminimizers on metric spaces, Illinois J. Math. 46 (2002), 383-403.

[5] - Approximation by regular sets in metric spaces, preprint, Linköping, 2004.

[6] J. Heinonen, Lectures on Analysis on Metric Spaces, Springer, New York, 2001.

[7] J. Heinonen, T. Kilpeläinen and O. Martio, Nonlinear Potential Theory of Degenerate Elliptic Equations, Oxford Univ. Press, Oxford, 1993.

[8] S. Keith, Modulus and the Poincaré inequality on metric measure spaces, Math. Z. 245 (2003), 255-292.

[9] S. Keith and X. Zhong, The Poincaré inequality is an open-ended condition, preprint, Jyväskylä, 2003.

[10] T. Kilpeläinen, J. Kinnunen and O. Martio, Sobolev spaces with zero boundary values on metric spaces, Potential Anal. 12 (2000), 233-247.

[11] J. Kinnunen and O. Martio, The Sobolev capacity on metric spaces, Ann. Acad. Sci. Fenn. Math. 21 (1996), 367-382.

[12] - - - Choquet property for the Sobolev capacity in metric spaces, in: Proceedings on Analysis and Geometry (Novosibirsk, Akademgorodok, 1999), Sobolev Institute Press, Novosibirsk, 2000, 285-290.

[13] - - - Nonlinear potential theory on metric spaces, Illinois Math. J. 46 (2002), $857-883$.

[14] —, - Potential theory of quasiminimizers, Ann. Acad. Sci. Fenn. Math. 28 (2003), 459-490.

[15] J. Kinnunen and N. Shanmugalingam, Regularity of quasi-minimizers on metric spaces, Manuscripta Math. 105 (2001), 401-423.

[16] P. Koskela and P. MacManus, Quasiconformal mappings and Sobolev spaces, Studia Math. 131 (1998), 1-17. 
A. Björn

[17] T. Laakso, Ahlfors $Q$-regular spaces with arbitrary $Q>1$ admitting weak Poincaré inequality, Geom. Funct. Anal. 10 (2000), 111-123.

[18] L. Saloff-Coste, Aspects of Sobolev-type Inequalities, London Math. Soc. Lecture Note Ser. 289, Cambridge Univ. Press, Cambridge, 2002.

[19] N. Shanmugalingam, Newtonian spaces: An extension of Sobolev spaces to metric measure spaces, Rev. Mat. Iberoamericana 16 (2000), 243-279.

[20] -, Harmonic functions on metric spaces, Illinois J. Math. 45 (2001), 1021-1050.

[21] - Some convergence results for p-harmonic functions on metric measure spaces, Proc. London Math. Soc. 87 (2003), 226-246.

Department of Mathematics

Linköpings universitet

SE-581 83 Linköping, Sweden

E-mail: anbjo@mai.liu.se

Received June 29, 2004

Final version January 21, 2005 